\title{
Video Article \\ A Photonic System for Generating Unconditional Polarization-Entangled Photons Based on Multiple Quantum Interference
}

\author{
Haruka Terashima ${ }^{1}$, Yoshiro Sato ${ }^{1}$, Satoshi Kobayashi ${ }^{1}$, Takaho Tsubakiyama ${ }^{1}$, Ryo Nozaki ${ }^{1}$, Satoshi Kubo ${ }^{1}$, Tomo Osada ${ }^{1}$, Kaoru Sanaka $^{1}$ \\ ${ }^{1}$ Department of Physics, Tokyo University of Science
}

Correspondence to: Kaoru Sanaka at sanaka@rs.tus.ac.jp

URL: https://www.jove.com/video/59705

DOI: doi:10.3791/59705

Keywords: Engineering, Issue 151, Polarization-entangled photons, parametric down-conversion, type-0, type-II, quantum interference, Sagnac interferometer, round-trip configuration

Date Published: 9/5/2019

Citation: Terashima, H., Sato, Y., Kobayashi, S., Tsubakiyama, T., Nozaki, R., Kubo, S., Osada, T., Sanaka, K. A Photonic System for Generating Unconditional Polarization-Entangled Photons Based on Multiple Quantum Interference. J. Vis. Exp. (151), e59705, doi:10.3791/59705 (2019).

\section{Abstract}

We present a high-performance source of unconditional polarization-entangled photons that have a high-emission rate, a broadband distribution, are degenerated and postselection free. The property of the source is based on the multiple quantum interference effect with a round-trip configuration of a Sagnac interferometer. The quantum interference effects make it possible to use the high generation efficiency of the polarization-entangled photons to process parametric down-conversion, and separate degenerated photon pairs into different optical modes without a postselection requirement. The principle of the optical system was described and experimentally used to measure the fidelity and Bell parameters, and also to characterize the generated polarization-entangled photons from a minimum of six combinations of polarization correlated data. The experimentally obtained fidelity and Bell parameters exceeded the classical local correlation limit and are clear evidence of the generation of unconditional polarization-entangled photons.

\section{Video Link}

The video component of this article can be found at https://www.jove.com/video/59705/

\section{Introduction}

The entangled state of photons has attracted considerable interest in the study of local realism in quantum theory and novel applications of quantum cryptography ${ }^{1}$, quantum dense coding ${ }^{2}$, quantum repeater ${ }^{3}$, and quantum teleportation ${ }^{4}$. Spontaneous parametric down-conversion (SPDC) is a second-order nonlinear process that has been introduced to directly produce entangled photon pairs in the polarization states. Owing to the recent development in quasi-phase-matching techniques, the periodically poled $\mathrm{KTiOPO}_{4}(\mathrm{ppKTP})$ and $\mathrm{LiNbO}{ }_{3}(\mathrm{ppLN})$ have become a standard technique ${ }^{5}$. Several types of entanglement sources are developed by combining these nonlinear crystals with a Sagnac interferometer $^{6,7,8}$. In particular, the scheme with orthogonally polarized photon pairs obtained by type-II SPDC makes it possible to generate unconditional polarization-entangled photons and also separate degenerated polarization-entangled photon pairs into different optical modes without postselective detection?

In contrast, type- 0 SPDC has the advantage of a simple setup and a high-emission ratio of photon pairs ${ }^{9}$. Moreover, the generated photon pairs in type-0 SPDC show a much broader bandwidth than the photons of type-II SPDC. The total photon-pair production rate per unit pump power is two orders of magnitude higher due to its large bandwidth ${ }^{8}$. A large bandwidth of correlated photon pairs allows a very short coincidence time between the detected photon pairs. This property has led to several potential applications such as quantum optical coherence tomography ${ }^{10}$, for achieving ultrashort temporal correlations through nonlinear interactions with the flux of entangled photons ${ }^{11}$, metrology methods using the very narrow dip in quantum interference ${ }^{12}$, quantum clock synchronization ${ }^{13}$, time-frequency entanglement measurement ${ }^{14}$, and multimode frequency entanglement ${ }^{15}$. However, the scheme with ordinary type-0 SPDC requires conditional detection schemes ${ }^{6}$ or wavelength filtering $^{8}$ or spatialmode filtering to separate the generated polarization-entangled photons ${ }^{16}$.

We realized a scheme that satisfies the properties of both type- 0 and type-II SPDC simultaneously based on multiple quantum interference processes ${ }^{17}$. The details of the optical system were described and experimentally used to measure the parameters that characterize the generated polarization-entangled photons using a minimum number of experimental data.

The Jones Vector of horizontal $(\mathrm{H})$ and vertical $(\mathrm{V})$ polarization state can be written as $|H\rangle \equiv\left(\begin{array}{l}0 \\ 1\end{array}\right)$ and $|V\rangle \equiv\left(\begin{array}{l}1 \\ 0\end{array}\right)$. All possible pure polarization states are constructed from coherent superpositions of these two polarization states. For example, the diagonal(D), anti-diagonal(A), right-circular(R), and left-circular(L) light, respectively, are represented by: 
$|D\rangle \equiv \frac{|H\rangle+|V\rangle}{\sqrt{2}}$,

$|A\rangle \equiv \frac{|H\rangle-|V\rangle}{\sqrt{2}}$,

$|R\rangle \equiv \frac{|H\rangle+i|V\rangle}{\sqrt{2}}$, and

$|L\rangle \equiv \frac{|H\rangle-i|V\rangle}{\sqrt{2}}$,

$\mathrm{H}$ and $\mathrm{V}$ are called the rectilinear polarization bases. $\mathrm{D}$ and $\mathrm{A}$ are called the diagonal polarization bases. $\mathrm{R}$ and $\mathrm{L}$ are called the circular polarization bases. These pure and also mixed states of the polarization can be represented by density matrixes based on the $\mathrm{H}$ - and $\mathrm{V}$ polarization bases ${ }^{18}$.

The operating principle of the scheme is shown in Figure 1a-e. The laser is injected into a polarization Sagnac interferometer comprised of a polarizing beam splitter (PBS), two half-wave plates set to $45^{\circ}$ (HWP1) and $22.5^{\circ}$ (HWP2), a ppKTP crystal, and mirrors. The polarization optics with this setup work for both the wavelength of the pump laser field and down-converted photons.

The $\mathrm{H}$-component of the pump laser passes through the PBS as shown in Figure 1a and round trips the setup in a clockwise (CW) direction. The polarization of the pump laser was inverted to the diagonal (D) state through HWP2. Here the V-component of the pump laser works for down-conversion, and the generated photons are V-polarized with type-0 SPDC. The SPDC polarization state of generated photon pairs can be represented as:

$\left|\psi_{\mathrm{a}}\right\rangle=|V V\rangle$

The down-converted photon pairs are H-polarized through the HWP1 set to $45^{\circ}$ as shown in Figure $\mathbf{1 b}$, and the polarization state becomes:

$\left|\psi_{\mathrm{b}}\right\rangle=|H H\rangle$

The pump laser beam again injected the inverted photon pairs into the ppKTP. The generated photon pairs from the second SPDC are both Vpolarized and superposed with the photon pairs generated by the first SPDC for a collinear optical mode as shown Figure 1c. The polarization state of the photon pairs after the second SPDC is represented as:

$\left|\psi_{\mathrm{c}}\right\rangle \propto|H H\rangle+e^{i \phi}|V V\rangle_{(4)}$

where $\phi$, is the relative phase between the photon pair from the first and second SPDC. The phase does not vary with time because it is determined by the HWP1's material dispersion between the pump laser and the down-converted photons, and adjustable by tilting HWP1. The H (V)-polarization state of the down-converted photons was inverted to A (D) state as shown in (1). The polarization state of the output photon pair from HWP2 is represented as:

$\left|\psi_{\mathrm{d}}\right\rangle \propto \sin \frac{\phi}{2}|H V\rangle+\frac{i}{\sqrt{2}} \cos \frac{\phi}{2}(|H H\rangle+|V V\rangle) \stackrel{\phi=\pi}{\longrightarrow}|H V\rangle$

When the phase $\phi=\pi$ is set by tilting HWP1, only the first term of the state (5) remains as shown in Figure 1d. This is the quantum interference process that corresponds to the reverse Hong-Ou-Mandel (HOM) interference process of the polarization bases ${ }^{19}$. When the $\mathrm{H}$ photon passes through PBS and the V-photon is reflected by PBS, the polarization state of the output photon pairs from PBS is represented as $|H\rangle_{1}|V\rangle_{2}$ for optical mode1 and 2 as shown in Figure 1e.

Conversely, the V-component of the pump laser was reflected by PBS as shown in Figure $1 \mathrm{f}$ and round tripped in a counter-clockwise (CCW) direction. Through similar multiple type-0 SPDC processes and unitary transformations, the polarization state of the output from PBS becomes $|V\rangle_{1}|H\rangle_{2}$. When the polarization state of the pump laser was prepared in diagonal (D) state, the relative phase between $\mathrm{H}$ - and $\mathrm{V}$-components of the pump laser was zero. Therefore, the output state of generated photons from CW and CCW directions are superposed with the same amplitudes and represented as:

$\left|\psi_{\text {oUT }}\right\rangle \propto|H\rangle_{1}|V\rangle_{2}+|V\rangle_{1}|H\rangle_{2}$. 
The output state is a polarization-entangled state known as one of the Bell states and can be converted to other three states using the polarization optics elements ${ }^{7}$. Using the relation shown in (1), the output state $\left|\psi_{\text {OUT }}\right\rangle$ can be represented by diagonal polarization bases as:

$|D\rangle_{1}|D\rangle_{2}-|A\rangle_{1}|A\rangle_{2}$ and by circular polarization bases as: $|R\rangle_{1}|R\rangle_{2}-|L\rangle_{1}|L\rangle_{2}$

\section{Protocol}

The adopted procedure comprises four main stages using the overall experimental setup shown in Figure 2. The first stage was the preparation of the pump laser for SPDC. In the second stage, the optical interferometer - Sagnac interferometer was constructed using a nonlinear crystal and optical polarization components. The coincidence measurement procedure using the electrical components shown in Figure $\mathbf{3}$ was described in the third stage. Finally, the actual photon correlation data shown in Figure $\mathbf{4}$ was used to estimate the fidelity and Bell parameters of the generated unconditional polarization-entangled photons.

\section{Configuration of the pump laser}

1. Switch on the $405 \mathrm{~nm}$ grating-stabilized single-frequency laser diode. Adjust the output power to a few mW by reducing the input electrical current to the laser diode and by neutral density filters.

2. Construct an external cavity between the surface of the laser diode and the holographic grating $\left(3,600 \mathrm{~mm}^{-1}\right)$ to realize a single-frequency operation referred to as a spectrometer. Place the holographic grating about $45^{\circ}$ against the laser diode surface and slowly move the screw to adjust the degree, and maximize the output power from the cavity by referring to the image of the beam.

3. Couple a laser to the polarization-maintaining optical fiber (PMF) to run a single spatial-mode operation. Adjust the fiber coupler screws to maximize the output power from PMF using a power meter.

4. Collimate the output laser from the PMF with a fiber coupler lens. Channel the output laser through an isolator into the center of half-wave plate (HWP), a quarter-wave plate (QWP), and a short-pass dichroic mirror (DM) as shown in Figure 2. For the purpose of generating the polarization-entangled photons with the state as in (6), set the polarization state of the pump laser with diagonal (D) by setting the HWP to $22.5^{\circ}$, and QWP to $0^{\circ}$.

\section{Construction of the interferometric setup}

1. Place a dichroic mirror (DM), a regular mirror, a PBS, and a ppKTP crystal with dimensions: $10 \mathrm{~mm}$ long (crystallographic $x$-axis), $10 \mathrm{~mm}$ wide ( $y$-axis), and $1 \mathrm{~mm}$ thick (z-axis) as shown in Figure 2. The PBS operates at both the wavelength of the laser (405 $\mathrm{nm})$ and that of the down-converted photons $(810 \mathrm{~nm})$. The poling period of the ppKTP crystal is $3.425 \mu \mathrm{m}$ which is designed for the collinear type-0 SPDC with $405 \mathrm{~nm}$ laser pump and has an anti-reflection coating at both wavelengths.

2. Adjust the PBS and mirrors using the pump laser $(405 \mathrm{~nm})$ and a reference laser $(810 \mathrm{~nm})$. Since the length from the input to output of the interferometer is about $600 \mathrm{~mm}$, make the transmitted and reflected light from PBS parallel for more than $600 \mathrm{~mm}$ (desirable for a few meters) to make spatial mode matchings.

3. Place HWP1 and HWP2 into the setup. They operate at both $405 \mathrm{~nm}$ and $810 \mathrm{~nm}$ wavelengths. Adjust the HWPs to be perpendicular to the incident light using the reflected light from the surface. Set the angle of HWP1 to $45^{\circ}$ and HWP2 to $22.5^{\circ}$

4. Place a retroreflector into the setup. Adjust the position of the retroreflector such that the clockwise (CW) and counter-clockwise (CCW) reference beams are on the same spatial mode. Place charge-coupled device (CCD) cameras on mode 1 and 2 in Figure 2 to refer the beam profiling images from the output of the interferometer. Adjust the mirror and retroreflector to make the spatial mode matching by referring the profiling images on the camera.

5. Place a focus lens between QWP for laser and DM. Since the length from the input to output of the interferometer is about $600 \mathrm{~mm}$, select a lens with a focus length of $300 \mathrm{~mm}$. Empirically set the focal point of the input laser pump to not be on the exact middle point of the interferometer but to be around the generation position of the second SPDC to make same-level generation efficiency of down-converted photons between first and second SPDC.

6. Remove the CCD camera and place QWPs, polarizers (POLs), interference filters (IFs) with an $810 \mathrm{~nm}$ center and $3 \mathrm{~nm}$ bandwidth in the mode 1 and 2 as shown in Figure 2. Adjust the optical elements to be perpendicular to the incident light using the reflected light. Couple the reference laser beams to the multimode fibers using fiber couplers for detection.

7. Place a $300 \mathrm{~mm}$ focus lens between DM and QWP in mode 1 and mode 2. Make the output reference laser beams to collimate for detection.

8. Connect the multimode fibers to the single-photon counting modules (SPCMs) constructed from Silicon (Si) avalanche photodiodes. Switch off the reference laser. Switch on the SPCMs in a darkroom condition, and count the down-converted photons.

9. Adjust the temperature of ppKTP crystal mounted on a temperature controller by referencing the count rates of down-converted photons. The appropriate temperature is typically $25-30^{\circ} \mathrm{C}$.

10. Adjust the tilting angle of HWP1 to maximize the count rates of down-converted photons. If the count rates are too weak, measure the counts without the optical elements in mode 1 and 2.

\section{Measurement procedure of the coincidence count}

1. Select the polarization bases in mode 1 and 2 to measure the incident polarization-entangled photons using POLs and QWPs as shown in Figure 3. For the measurement of the incident photon with $\mathrm{H}(\mathrm{V})$ base, set the QWP to $0^{\circ}$ and the $\mathrm{POL}$ to $0^{\circ}\left(90^{\circ}\right)$. For the measurement of the incident photon with $D(A)$ base, set the QWP to $0^{\circ}$ and the POL to $45^{\circ}\left(-45^{\circ}\right)$. For the measurement of the incident photon with $R(L)$ base, set the QWP to $45^{\circ}\left(-45^{\circ}\right)$ and the POL to $0^{\circ}$.

2. Connect the transistor-transistor logic (TTL) signal generated from the SPCM in mode 2 to the start signal input of a time-to-amplitude converter (TAC), and the signal in mode 1 to the stop signal input after it has passed through the electrical delay line (Delay). TAC generates electrical signals from 0 to $10 \mathrm{~V}$ corresponding to the time delay between two signals. 
1. In this experiment, set the time delay $\Delta T$ as $50 \mathrm{~ns}$ by selecting the delay line pins. Set the display of $\mathrm{PC}$ to show $100 \mathrm{~ns}$ time range by setting the dial of TAC. Then TAC generates $5 \mathrm{~V}$ signals as $50 \mathrm{~ns}$ delay time given by the electrical delay line. Therefore the $5 \mathrm{~V}$ signals correspond to the coincidences at 0 ns delay time of actual pulses coming from SPCMs. The coincidences at 0 ns delay time appear in the center of the display time range as shown in Figure 3.

3. Click the start button of the software, called MAESTRO-32, to measure the pulse height distribution and record the distribution with a computer controlled (PC) multi-channel analyzer (MCA). In this experiment, set the measurement time of TAC for $30 \mathrm{~s}$. Analyze the height distribution of the TAC pulses from 0 to $10 \mathrm{~V}$ which corresponded to a -50 to $50 \mathrm{~ns}$ delay time between the incident photons and the SPCMs by the setting described in step 3.2.

4. After recording the pulse height distribution, obtain the pulse height distribution data for several polarization bases as shown in Figure 4. Select the time window to be considered for coincidence counts for the analyzation of the data. Since the width of the pulse peak is determined by the SPCM's resolution time of $1 \mathrm{~ns}$, the coincidence time window is necessary to be larger than the resolution time.

1. In this experiment, choose the coincidence time window to be $10 \mathrm{~ns}$. Estimate the coincidence counts by integrating the area of the time window.

\section{Estimation procedure of the Fidelity and Bell parameters}

1. Determine the polarized second-order correlations $g_{x x}^{(2)}(0)$ and cross-polarized second-order correlations $g_{x \bar{x}}^{(2)}(0)$, where $x$ refers to the polarization states $\mathrm{H}, \mathrm{D}$, and $\mathrm{R}$, and $\bar{x}$ refers to the cross-polarization states $\mathrm{V}, \mathrm{A}$, and $\mathrm{L}$. Obtain these functions by dividing the measured coincidence counts $R_{\mathrm{CC}}$ by the background level $R_{\mathrm{back}}$. Figure 4 shows the actually measured pulse height distribution of coincidence counts with several polarization bases for 30 s.

NOTE: For example, the coincidence counts the with polarization base $\mathrm{HH}$ gives $R_{\mathrm{CC}}=13.0 \pm 3.6$ count/30 s for coincidence window 10 ns. The average back ground level for the coincidence window is calculated as 4.3 count $/ 30 \mathrm{~s}$. Since second-order correlations are given by $R_{\mathrm{CC}} / R_{\text {back }}$, the polarized second-order correlation functions with polarization base $\mathrm{HH}$ becomes $g_{H H}^{(2)}(0)=3.1 \pm 0.8$. Similarly secondorder correlation functions with other polarization bases are given as: $g_{D D}^{(2)}(0)=95.3 \pm 4.0$, and $g_{R R}^{(2)}(0)=89.8 \pm 3.7$ and crosspolarized second-order correlation functions as: $g_{H V}^{(2)}(0)=102.9 \pm 4.4, g_{D A}^{(2)}(0)=11.4 \pm 1.3$ and $g_{R L}^{(2)}(0)=16.4 \pm 1.5$.

2. Determine the degree of polarization correlation between two photons for three polarization bases defined by ${ }^{20,21}$ :

$C_{\mathrm{X}}=\frac{g_{x x}^{(2)}(0)-g_{x \bar{x}}^{(2)}(0)}{g_{x x}^{(2)}(0)+g_{x \bar{x}}^{(2)}(0)}$ (7)

where $X$ refers to the polarization bases of the rectilinear $(H$ and $V)$, diagonal $(D$ and $A)$, and circular $(R$ and $L)$ bases. The measured secondorder correlation functions give the degree of each polarization bases as follows: $C_{\text {rectilinear }}=-0.94 \pm 0.02, C_{\text {diagonal }}=0.79 \pm 0.02$, and $C_{\text {circular }}=0.69 \pm 0.03$.

3. Determine the fidelity of generated entangled photons. Calculate the fidelity of the polarization-entangled state with respect to the state (6) in three bases ${ }^{20,21}$ :

$f=\frac{1-C_{\text {rectilinear }}+C_{\text {diagonal }}+C_{\text {circular }}}{4}$

The measured degrees of polarization correlation was $f=0.85 \pm 0.01$. The number exceeded the classical polarization correlation limit of 0.50 .

4. Determine the Bell parameters of the generated entangled photons ${ }^{21}$. Calculate the parameters from the polarization correlations as follows 19,20 .

$S_{\mathrm{RC}}=\sqrt{2}\left(1-C_{\text {rectilinear }}+C_{\text {circular }}\right)$

$S_{\mathrm{DC}}=\sqrt{2}\left(1+C_{\text {diagonal }}+C_{\text {circular }}\right)$

$S_{\mathrm{RD}}=\sqrt{2}\left(1-C_{\text {rectilinear }}+C_{\text {diagonal }}\right)$

The measured bases of polarization correlation were $S_{\mathrm{RC}}=2.31 \pm 0.04, S_{\mathrm{DC}}=2.09 \pm 0.05, S_{\mathrm{RD}}=2.44 \pm 0.04$. These

numbers exceed the classical parameter limit of 2 and violate the Bell inequality. 


\section{Representative Results}

The optical system to generate unconditional entangled photons for polarization states based on multiple quantum interferences and detection schemes to estimate the experimental fidelity by polarization correlation of generated photon pairs was discussed. The estimated fidelity of the generated photons exceeded the classical local correlation limit of 0.50 . The measured Bell parameters exceeded the classical parameter limit of 2 and violated the Bell inequality. In this paper, coincidence measurements obtained from a minimum of six combinations of polarization bases were used to evaluate these parameters. Furthermore, it is possible to completely reconstruct the density matrix of the generated polarizationentangled photons via quantum state tomography, which requires coincidence measurements of 16 combinations of polarization bases ${ }^{18}$.

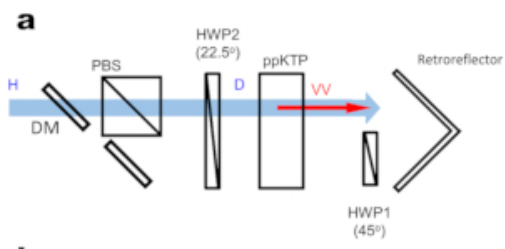

b

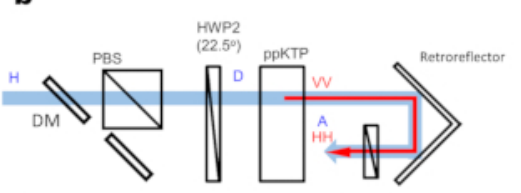

C

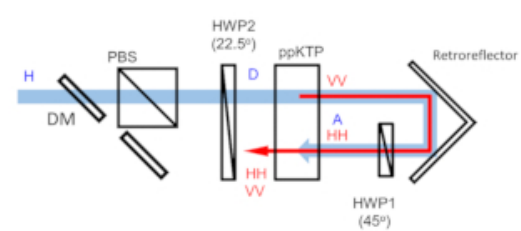

d
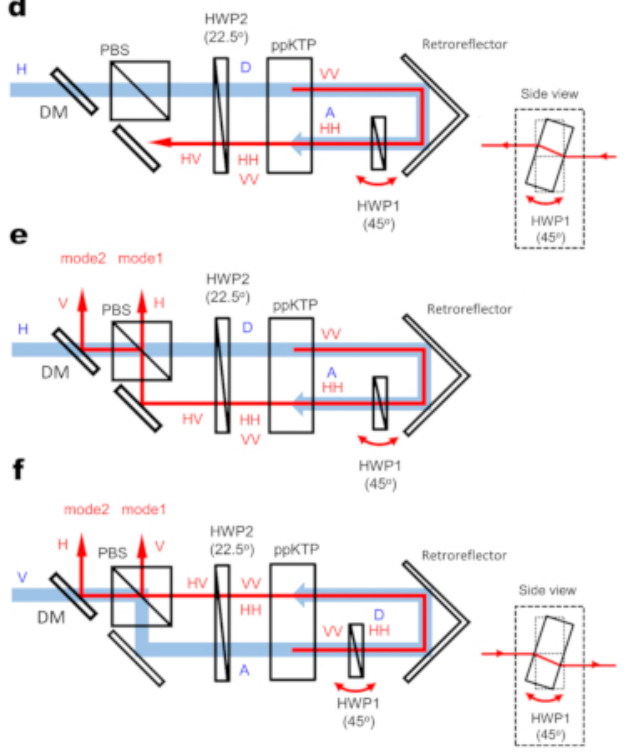

Figure 1: Schematic of an integrated double-pass polarization Sagnac interferometer. (a) The generation of photon pairs after the first spontaneous parametric down-conversion (SPDC). (b) Polarization rotation of the photon pairs by a half-wave plate (HWP1). (c) The generation of photon pairs after the second SPDC. (d) The quantum interference between photon pairs of the first and second SPDC by HWP2. (e) Output photon pairs produced in the clockwise (CW) direction. (f) Output photon pairs produced in the counter-clockwise (CCW) direction. Please click here to view a larger version of this figure.

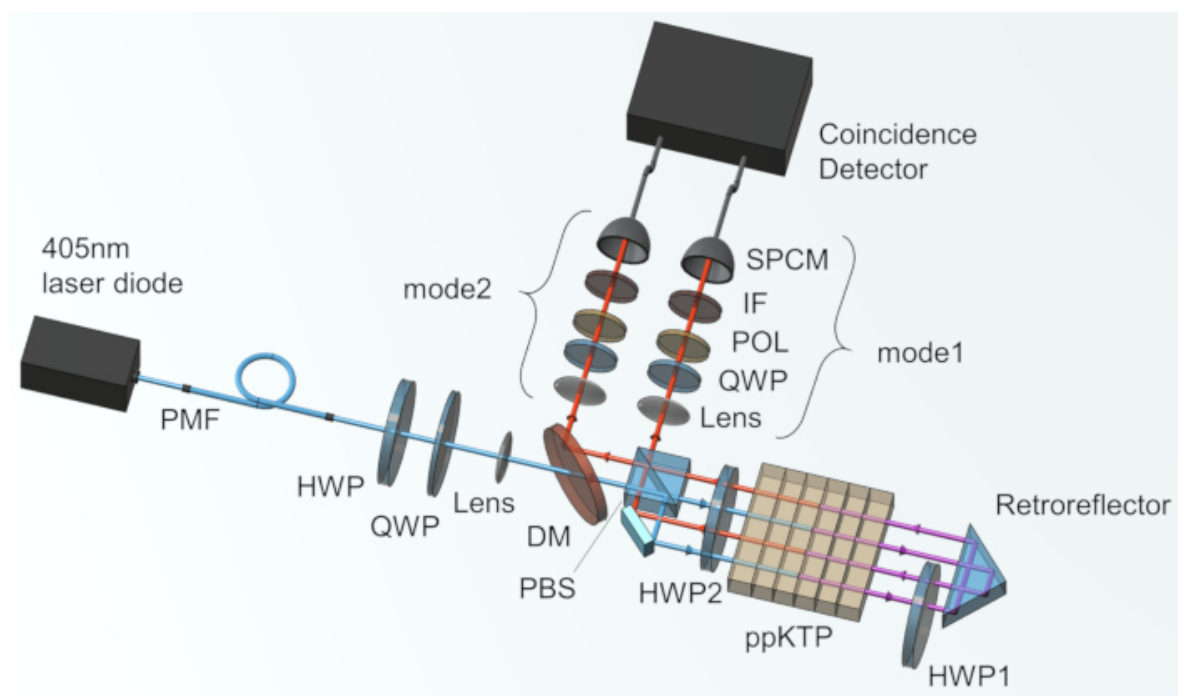

Figure 2:Overall optical system for generating unconditional polarization-entangled photons. The first half-wave plate (HWP) and a quarter-wave plate (QWP) are used to set the polarization state of the pump laser passing through polarization-maintaining optical fiber (PMF). The output photons were passed through lenses, QWPs, polarizers (POLs), and interference filters (IFs) in modes 1 and 2 , and detected by the single-photon counting modules (SPCM). Please click here to view a larger version of this figure. 


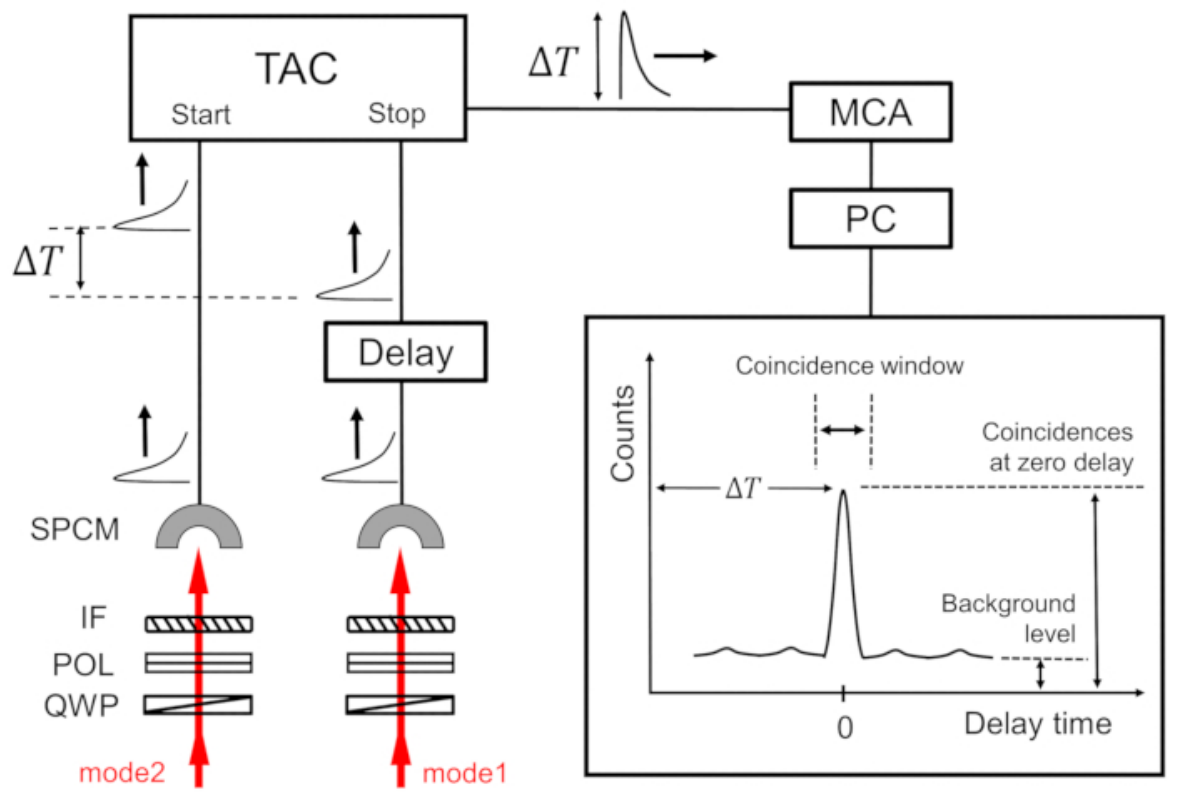

Figure 3: Overall coincidence detection system for the generated polarization-entangled photons. The electrical signals from the SPCM were used to start and stop the signal of the time-to-amplitude converter (TAC) through an electrical delay line (Delay). The pulse height distribution obtained from time difference was analyzed with a computer controlled (PC) multi-channel analyzer (MCA). Please click here to view a larger version of this figure.

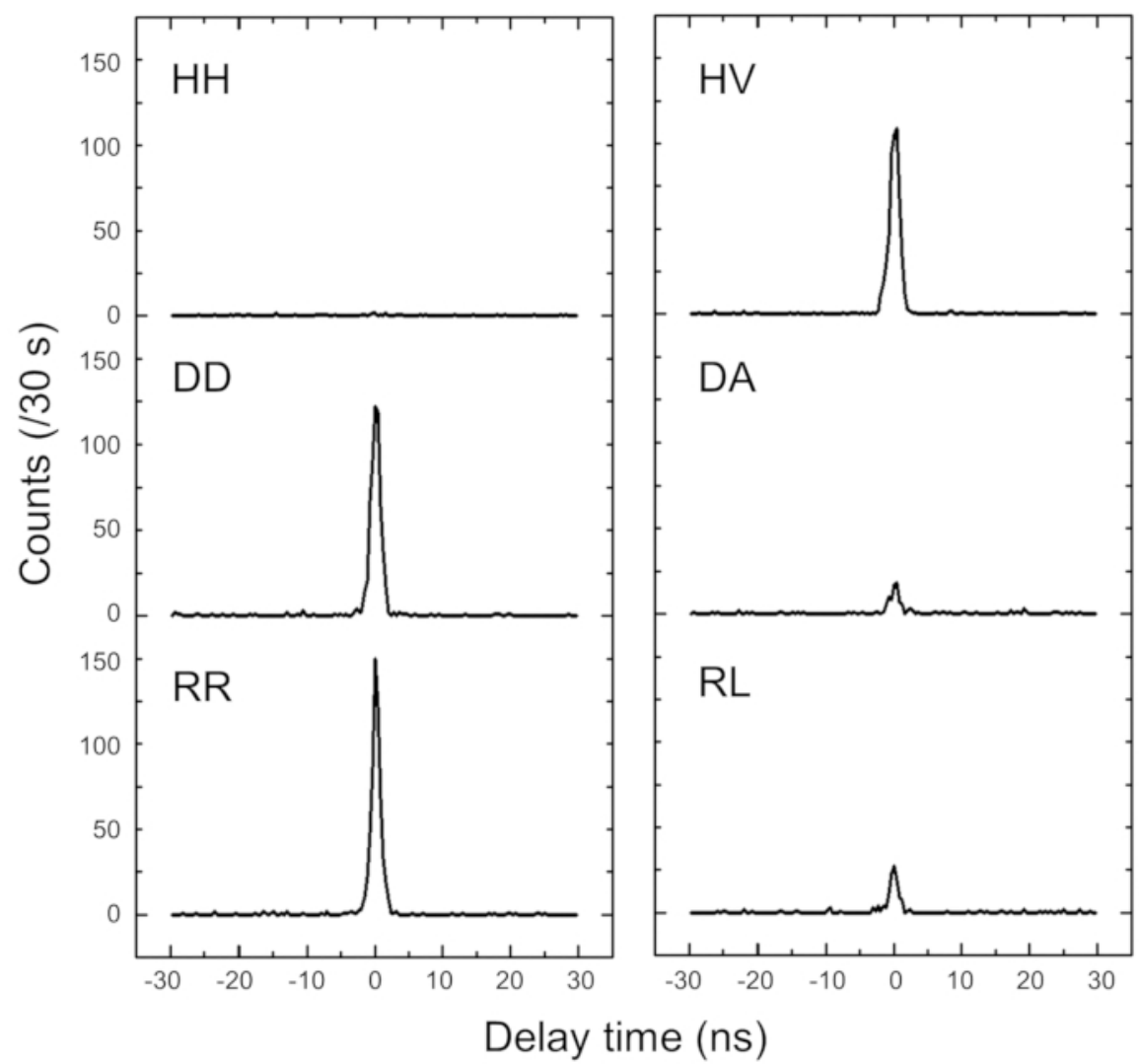

Figure 4: Measured time difference distributions with parallel and orthogonal polarizer settings. The combinations are horizontal $(H)$, vertical $(\mathrm{V})$, diagonal $(\mathrm{D})$, anti-diagonal $(A)$, right-circular $(\mathrm{R})$, and left-circular $(\mathrm{L})$ polarization bases. Please click here to view a larger version of this figure. 


\section{Discussion}

The critical step within the protocol is how to maximize the fidelity of the generated polarization entangled photons. The estimated fidelity and Bell parameters are currently limited, mainly because we used multimode fibers to collect the generated entangled photons. The tilting of HWP1 affected the height difference of the spatial modes between the photons of the first and second SPDC and caused a spatial-mode mismatch on the output of the Sagnac interferometer. The fidelity is expected to be higher when using single-mode fibers that filter out the spatial-modeoverlapping area of the generated first and second SPDC photons. Moreover, the birefringence effect of the ppKTP crystal affected the mode mismatch between the first and second SPDC photons. In future, we can possibly improve the parameters by using additional compensation crystals.

The significance of the protocol is to realize several properties simultaneously with respect to existing method. The source of the polarization entangled photons with the protocol have a high-emission rate, are degenerate, have a broadband distribution, and are post-selection free. The characteristic advantage of the protocol is based on the multiple quantum interference using a double-pass polarization Sagnac interferometer. The photonic system makes it possible to use the large generation efficiency of polarization entangled photons and to separate degenerate photon pairs into different optical modes with no requirement of postselection. The system of high-performance polarization entangled photons can be applied for novel photonic quantum information technologies ${ }^{1,2,3,4}$.

\section{Disclosures}

The authors have nothing to disclose.

\section{Acknowledgments}

This research was supported by Research Foundation for Opto-Science and Technology, Japan. We thank to Dr. Tomo Osada for the useful discussions.

\section{References}

1. Ekert, A.K. et al. Quantum cryptography based on Bell's theorem. Physical Review Letters. 67, 661-663 (1991).

2. Mattle, K., Weinfurter, H., Kwiat, P.G., Zeilinger, A. dense coding in experimental quantum communication. Physical Review Letters. 76, 4656-4659 (1996).

3. Pan, J.-W., Bouwmeester, D., Weinfurter, H., Zeilinger, A. experimental entanglement swapping: entangling photons that never interacted. Physical Review Letters. 80, 3891-3894 (1998).

4. Bouwmeester, D. et al. Experimental quantum teleportation. Nature. 390, 575-579 (1997).

5. Armstrong, D.J., Alford, W.J., Raymond, T.D., Smith, A.V. Absolute measurement of the effective nonlinearities of KTP and BBO crystals by optical parametric amplification. Applied Optics. 35, 2032-2040 (1996).

6. Shi, B.-S., Tomita, A. Generation of a pulsed polarization entangled photon pair using a Sagnac interferometer. Physical Review A. 69, 013803 (2004).

7. Kim, T., Fiorentino, M., Wong, F.N.C. Phase-stable source of polarization-entangled photons using a polarization Sagnac interferometer. Physical Review A. 73, 012316 (2006).

8. Steinlechner, F. et al. Efficient heralding of polarization-entangled photons from type-0 and type-II spontaneous parametric downconversion in periodically poled $\mathrm{KTiOPO}_{4}$. Journal of the Optical Society of America B. 31, 2068 (2014).

9. Steinlechner, F. et al. Phase-stable source of polarization-entangled photons in a linear double-pass configuration. Optics Express. 21, 11943-11951 (2013).

10. Okano, M. et al. 0.54 resolution two-photon interference with dispersion cancellation for quantum optical coherence tomography. Scientific Reports. 5, 18042 (2015).

11. Dayan, B., Pe'er, A., Friesem, A.A., Silberberg, Y. Nonlinear interactions with an ultrahigh flux of broadband entangled photons. Physical Review Letters. 94, 043602 (2005).

12. Nasr, M. B. et al. Ultrabroadband biphotons generated via chirped quasi-phase-matched optical parametric down-conversion. Physical Review Letters. 100, 183601 (2008).

13. Giovannetti, V., Lloyd, S., Maccone, L., Wong, F.N.C. Clock synchronization with dispersion cancellation. Physical Review Letters. 87, 117902 (2001).

14. Hofmann, H. F., Ren, C. Direct observation of temporal coherence by weak projective measurements of photon arrival time. Physical Review Letters A. 87, 062109 (2013).

15. Mikhailova, Y. M., Volkov, P. A., Fedorov, M. V. Biphoton wave packets in parametric down-conversion: Spectral and temporal structure and degree of entanglement. Physical Review A. 78, 062327 (2008).

16. Jabir, M. V., Samanta, G. K. Robust, high brightness, degenerate entangled photon source at room temperature. Scientific Reports. 7, 12613 (2017).

17. Terashima, H., Kobayashi, S., Tsubakiyama, T., Sanaka, K. Quantum interferometric generation of polarization entangled photons. Scientific Reports. 8, 15733 (2018).

18. Altepeter, J.B., Jeffrey, E.R. Kwiat, P.G. Photonic state tomography. Advances In Atomic, Molecular, and Optical Physics. 52, 105-159 (2005).

19. Hong, C K., Ou, Z.Y., Mandel, L. Measurement of subpicosecond time intervals between two photons by interference. Physical Review Letters. 59, 2044-2046 (1987).

20. Hudson, A.J. et al. Coherence of an Entangled Exciton-Photon State, Physical Review Letters. 99, 266802 (2007). 
21. Young, R.J. et al. Bell-Inequality Violation with a Triggered Photon-Pair Source Physical Review Letters. 102, 030406 (2009). 